\title{
Laparotomía exploradora en la enfermedad de Hodgkin
}

\author{
Drs. M. Varela*, A del Río", H. del Pozo**, Y. Beresi**
}

En los últimos años ha variado fundamentalmente el concepto de Enfermedad de Hodgkin (E. H.) : se la considera ahora como potencialmente curable en un porcentaje de casos cada día mayor.

Para selecionar el tratamiento adecuado es indispensable establecer claramente la extensión o "clasificación" de la enfermedad. Listo cs aún más importante en niños que en adultos, ya que en esa cdad los efectos nocivos de una Radioternpia extensa y de una Qui. mioterapia prolongada con varias drogas son de mayor significación. En los niños, después de la radioterapia se pueden producir retardos de crecimiento, deformaciones del esqueleto y neoplasias secundarias: de alle lit importancia de delinear la extensión de la enfermedad y definir los límites apropiados para las iпrdiaciones. La quimioterapia prolongada puede producir azoospermia, daño vesical y otras complicaciones a largo plazo aún desconocidas (1).

Hay suficiente información como para estimar que el estudio clínico habitual no basta para establecer una clasificación correcta, sino que esta debe ser corroborada o modificada por una Iaparotomía exploradora que permita practicar esplenectomla, biopsias liepáticas, ganglionares y de médula ósea $(1,4,6,7,9$, 11).

"Scrv. Citugía. Iosp., Calvo Mackenna.

* Depto. Oncologia, Hosp. Calvo Mackenna.
Este trabajo representa la experiencia retrospectiva del Departamento de Oncologia del Hospital Luis Calvo Mackenna desde di. ciembre de 1967 hasta septicmbre de 1975. El Departamento cuenta con un equipo formado por pediatras, cirujanos, radiologos, histopatólogos, hematólogos, radioterapeutas (FALP) y psiquiatras.

En este Departamento se han reunido 253 tumores sólidos en niños (excluido S.N.G.), 95 de ellos linfomas y de estos $40 \mathrm{E}$. H.

Se sigue la clasificación clínica de Ann Ar. bor y la histopatologia de Rye.

\section{MATERIAL Y METODO}

Revisamos 40 casos de E. H., 32 hombres y 8 mujeres, 26 de ellos entre los 3 y los 7 años, con edad menor de 2 años 8 meses y mayor de 13 años 9 meses al iniciar su enfermedad. El estudio y clasificacion clínicos se hizo siguiendo los puntos cstablecidos por el Departamento (3): anamnesis y examen físico completo, exámencs de laboratorio (hejnograma, V.H.S., batería inmunológica, PPD, etc.), radiografia de tórax, biopsia ganglionar, linfo. grafia y/o laparotomia.

La linfografía es un método áliji y practicado en la mayoria de los centros especializados; su técnica en el niño es algo engorrosa, requiere anestesia general y la interpretación radiológica es más difícil que en el adulto, no muestra el compromiso del hígado ni del bazo, ni de los ganglios periaórticos superiores, del 
lijio espléenico " mesentécicos. No sttbstituye a la laparotomíl, pero puede facilitar al cirujano la búsqueda y localización de ganglios sospechosos con ayuda de radiografias transoperatorias. En nuestro Departamento se practicaron 19 linfografías liasta 1971 , después ha sido reemplazada por la laparotomía por las dificultades señaladas y por problemas pari obtener el materiaI necesario (agujas, coloran. tes, medio de contraste) .

Filler y Col. (4) presentan los sifuientes resultados en $\mathbf{5 5}$ linfografías:

$\begin{array}{ccc}\text { Linfografia } & \begin{array}{c}\text { Confirmación Histológica } \\ \text { Positiva }\end{array} \\ \text { Negativa }\end{array}$

Estos resultados indicarjan que, con suficiente experiencia, las linfografías interpretadas como dudosas o negativas tienen un valor orientador indudable ya que la histología los confirma como negativas en un alto porcentaje $(97,5 \%)$.

Estimamos que en el futuro deluemos reiniciar el uso sistemático de la linfografía. Como el medio de contraste persiste varios meses, permite seguir durante ese tiempo la evolución cle la enfermedad, y puede ser de especial nuilidad en los grarlos I A, ya que de resultar negativa se puede evitar o postergar la laparotomía.

La cintigrafía, aunque de menor rendimiento, puede laacerse en niños muy pequeños en Ios cuales la linfografía no pucle efectuarse (1).

\section{LAPAROTOAIA EXPLORADORA}

La primera laparotomía exploradora la efectuamos en 1969, el mismo año en que fue preconizada por Glatstein y Col, (7). Antes habian consultado 10 enfermos. De los 30 posteriores a 1969 fueron operados 28. En los dos restantes no se hizo por ser uno Grado Iv $B$ y el otro i $A$ en control y remisión completa durante 3 años.

En el período 1969 a 1971 transcurrian meses o años desde la primera consulta hasta lecidir la operación; en 1975 , en 6 casos, tenemos un promedio de 12 días desde la primera consulta hasta la intervención.

La lapirotomía debe seguir las siguientes pautas:

1. Esplenectomía ción.

2. Biopsias hepáticas por sección y por pun-

3. Biopsias ganglionares siguiendo rutina establecida.

4. Biopsia méclula ósea (cresta ilíaca o costilla).

5. Ooforopexia (li) para protección con. tra radioterapia, en línea media y por detrás del útero (incisión Pfannenstiel).

\section{Esplenectomia}

Se practicó en 22 casos: 12 de ellos tenían E. H., 9 eran normales y uno TBC. En las primeras laparotomías efectuadas en el Hospital, el cirujano determinaba o no la extirpación del bazo según su tamaño y aspecto macroscópico, ahora se practica de rutina. Iat atsencia de esplenomemalia no es fndice de indemnidad: 8 casos sin esplenomegalia clinica fueron positivos de E. H.; por $\epsilon \mathbf{l}$ contrario los 4 casos con esplenomegalia clínica fueron todos positivos.

No hubo complicaciones inmediatas, salvo una infección de herida operatoria. No sc apreció aumento significativo del número de plaquetas.

Cono complicación alejada, y que podria relacionarse con la esplenectomía, aparece una caso de una niña de 11 años que dos años clespućs de la operación presentó una meningitis neumocócica que evolucionó bien. Hubo (los fallecidos: una niña de 14 años en tratamiento con COPP y RT falleció 1 año des. pués por aplasia medular; un niño de 7 años falleció a los 7 meses con infiltración tumoral de pulmón y esófago y meningitis terminal. Los tres casos eran Grado ul $\mathrm{B}$.

Varios niños presentaron herpes zóster y varicela grave, que son complicaciones frecuentes de la enfermedad y no atribuilbles a la esplenectomía: hay 6 herpes zóster en los 40 
enfermos, is en 22 esplenectomíls y 3 en 18 no esplenectomizados.

El problema de las infecciones masivás postesplancctomía es significativo y se han desrrito mucrtes relacionadas con ella. Hay que recorclat que en muchos de los casos publicalos st: usaba quimioterapia con COPP o MOPP que son drogas fuertementc inmunosupresomias. Se ha descrito (5) que las complicuciones alejaclas de Ia esplenectomía depenclen de la enfermedad causal (talasemia, linforna) y cle Ia edad: la mitad de las muertes ocurrieron en el grupo de menores de 2 años, que forman solo el $15 \%$ del total de 1419 esplenectomfas revisadas.

El promedio de edad de nuestros enfermos fue de 8 años y todos mayores de 4 años.

Estimamos que sobre los 4 años todo niño con E. H. debe ser esplenectomizado: además de la nejor información para efectuar la tcrapia adccuada, al liacer innecesaria la irradiación del bazo, evita los efectos de ésta sobre el riñón y base del pulmón izquierdo.

\section{Biopsias}

ia) Hepática: se efectuó en 16 enfermos tomando una soja muestra, ninguno mostró compromiso histológico. Se recomiencla ahora hacer biopsias múltiples en ambos lóbulos, por sección y por punción.

b) Ganglionaver: se tomaron diferentes ganglios sospechosos en 24 casos y 15 fueron positivos. Es necesario seguir una rutina precstablecida y tomar, además de los ganglios sospechosos, muestras de grupos ganglionares específicos (periaórticos superiores e inferiores, hilio esplénico, mesentéricos, ilíacos) (2) . Los sitios de biopsia deben ser marcados con clips metálicos para permitir el control radiográ. fico y facilitar la radioterapia.

c) Médula Osea: se practicó en cresta ilíaca (o en undécirna costilla izquierda) en 12 casos: solo uno fue positivo, lo que coincide con el bajo rendimiento señalado por otros autores. El carácter focal de la localización medular de la E. $H$. ha hecho que se recomiende hoy día el estudio radiológico del esqucleco y cintigrafias (Ga fit) para prac. ticar la biopsia directamente en las zonas que aparezcan sospechosas.

\section{CAMBIO DE CLASIFICACION DESPUES} IDF LA LAPAROTOMIA

Tal vez lo más importante de esta revisión sea que la Iaparotomía exploradora, al revelar o no compromiso histológico bajo el diafrag. ma y/o de órganos extra linfáticos, hizo modificar la clasificación clínica pre-operatoria en un número importante de enfermos: de los 28 niños Iaparotomizados 11 debieron ser reclasificados en otros grados $=39 \%$.

Cambio de Grado

\begin{tabular}{rcr}
\hline preop. & Grado & postop. \\
\hline 8 & I & 6 \\
10 & II & 4 \\
9 & III & 16 \\
1 & IV & 2 \\
\hline
\end{tabular}

El sentido en que se produjo la variación fue el siguiente:

\begin{tabular}{rrrrr}
\hline 2 & grado & I & pasiron a grado & III \\
6 & grado & II & [pasaron a grado & III \\
2 & giado & III & pasitron a grado & JV \\
1 & grado & IV & pasaron a grado & III \\
\hline
\end{tabular}

La variación puede ser hacia Grados más avanzados o a menos avanzados, siendo lo primero lo más frecuente, 10 de 11 casos. La mayoría de los cambios se produjeron hacia el Grado in (9 casos) y sólo 2 fueron reclasificados del Grado in al rv.

Con respecto al tiempo transcurrido desde la iniciación de la enfermedad y el momento de Ia operación hemos visto que de 16 enfermos con más de $I$ año de evolución variaron su clasificación $8(50 \%)$; en cambio de 12 enfermos con menos de 1 año de evolución variaron sólo $3 \quad(25 \%)$.

En relación a ausencia o presencia de síntomas sistémicos, (pérdida inexplicada de más del $\mathrm{I} 0 \%$ del peso, fiebre sobre $38^{\circ}$, decaimien- 
to y palidez), o sea (Grados A o B, la diferen. cia es poco signiticativa: de 12 Grados A cambiaron $4(33 \%)$, de 16 Grados B cambiaron $7(43 \%)$.

No se apreció relación entre el tipo histológico y las variaciones de Grado. De 23 hombres cambiaron $7(30 \%)$, de 5 mujeres cambiaron $4(80 \%)$.

\section{RESUMEN}

Se presenta la experiencia deI Departamento de Oncología del Hospital L. Calvo Mackenna sobre laparotomía exploradora en la clasificación de la Enfermedad de Hodgkin y se comenta su utilidad.

Se analizan 40 E.H. en los cuales se practicaron 28 laparotomías descle 1969 a 1975 ; de los 28 casos operados 11 debieron ser reclasificados: $39 \%$. De estos 11 cambios 10 lo hicieron hacia Grados más avanzados.

En las 28 laparotomías se practicaron 22 esplenectomias, todos mayores de 4 años, y 12 fueron positivas de E. H.: $\mathbf{5 4 , 5 \%}$.

La ausencia de esplenomegalia clínica no es indice de indemnidad: 8 enfermos sin csplenomegalia fueron histológicamente positivos. Todos los cnfermos con esplenomegalia fueron positivos.

No hubo complicaciones inmediatas. No se observó diferencia en la evolución de la enfermedad ni en sus complicaciones entre los grupos no esplenectomizados y aquellos en que se realizó la esplenectomia.

Se analiza el resultado $y$ rendimiento de las biopsias hepáticas, ganglionares y de médula ósea, y se comenta la conducta a seguir en el futuro.

La linfografía se practicó en 13 cáos con resultados poco significativos, pero se estima que se debe insistir en ella, ya que con experiencia su rendimiento es importante.

\section{CONGLUSIONES}

La laparotomía exploradora es útil en la cla sificación de la Enfermedad de Hodgkin.

La laparotomía explotadora es un método de diagnóstico y de clasificación, por lo tanto sólo se justifica cuando se practica con un riesgo quírúrgico mínimo, y como una parte de un programa cuidadoso desarrollado en un Centro Oncológico que cuente con todos los medios para hacer la Radioterapia y Quimioterapia adecuadas.

\section{SUMMARY}

A revision of the experience with the use of Diagnostic Laparatomy in Hodgkin's disease of the Luis Calvo Mackenna Oncology Center is presented. The advantages of a better clasification of the disease are stressed.

40 pations with Hodgkin's disease are included, 28 of which had Laparatomy from 1969. to 1975, Il of the 28 had to be reclassified. 10 out of these it changex in classification to a more advance Grade.

Of the 28 patiens who had Laparotomy, Splenectomy was perfomed in 22, all of these were 4 years and older. Hodgkin's disease was present in 12 cases: $54,5 \%$.

The lack of palpable spleen does not cxclude its compromise: 8 patients with no palpable splcen had histologic evidence of Hodgkin's disease. All patients with palpable spleen had H. D.

There were no carly complications. One Splenectomizcd patient had pneumococcial meningitis 2 years after surgery and recovered eventfully.

The results and usefullness of liver, lymph node and bone narrow biopsy is commented elaborating a management program for the fuiture.

13 cases under went lymphography which was of little value due to the poor previous experience. It is hoped that this technique will be more useful in the future in our hands.

\section{REFERENCIAS}

I. Hays D. M.: The Staging of Hodgkin's Disease in Children Revicwed. Cancer, 35: 973, maro 75.

2. Hays M. D, et al.: Laparotomy for the: Staging of Hodgkin's Disease in Childrcn. J. Peliatr. Surg. 7. 517-527, 1972 . 
3. Lrget W. y Col: Exprricncia sobie enfermedad de Hodgkin en la pritnera decada de la vida. Rev. Chilena Perl. 45: 9, 1974 .

4. Filer R. M. et al: Experience with Clinical and Opcrative Staging of Hodgkin's Disease in Children. Journal Pediatric Surgery, 10: 321. Junio 1975.

5. Eraklis A. J. et al: Splenectomy in Children. A. Review of 1413 cases. Journal Pediatric Surgery, 7: 982,1972 .

6. Lowenbraug et al: Jiagnostic I aparotomy and Splenectomy for Staging Hodgkin's Disease. Annals Int. Med. 72: $655,1970$.

7. Glatstein ec al: The Value of Laparotomy and
Spienectomy in the Staging of Hulgkin's Disease. Cancer: 24, 709, Octubre 1969.

8. Aiscunberg et al: Spleen Involvement at the Onset of Hodgkin's Discase. Annals Int. Med. 74: 544, 1971 .

9. roung $R$. et al: Hougkin's Disease in Children. Blood, 42: 163, 1973.

10. Johnson R. E.: Is Staging Laparotomy Routinely Indicated in Hodgkin's Diserse. Annals Int. Med. 75: 459,1971 .

11. Jenkin R. T. D, et al: Hodgkin's Disease in Children. A. Retrospective Analysis: 1958-197\$. Cancer. 35: 979, matzo 1975 . 